\title{
In vitro anthelmintic, antibacterial and cytotoxic effects of extracts from plants used in South African ethnoveterinary medicine
}

\author{
L.J. McGaw ${ }^{a}$, D. Van der Merwe ${ }^{b, 1}$ and J.N. Eloff ${ }^{a}$

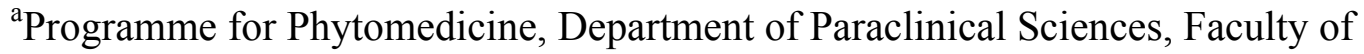 \\ Veterinary Science, University of Pretoria, Private Bag X04, Onderstepoort 0110 , \\ Pretoria, South Africa \\ ${ }^{\mathrm{b}}$ Animal Health for Developing Farmers Division, ARC-Onderstepoort Veterinary \\ Institute, Private Bag X05, Onderstepoort 0110, Pretoria, South Africa
}

\begin{abstract}
Many plants are used for ethnoveterinary purposes in South Africa, particularly in rural areas. Extracts of 17 plant species employed to treat infectious diseases were prepared using three solvents and the antibacterial activity of the extracts was determined against two Gram-positive and two Gram-negative bacteria. Anthelmintic activity was evaluated against the free-living nematode Caenorhabditis elegans and toxicity was determined using the brine shrimp larval mortality test. Most of the plant extracts demonstrated antibacterial activity, with the best minimum inhibitory concentration (MIC) being $0.1 \mathrm{mg} \mathrm{mL}^{-1}$. More than a third of the extracts displayed anthelmintic activity. Toxic effects against brine shrimp larvae were shown by $30 \%$ of extracts, with the lowest $\mathrm{LC}_{50}$ recorded as $0.6 \mathrm{mg} \mathrm{mL}^{-1}$. The promising biological activity displayed by a number of plant extracts supports the ethnoveterinary use of these plants but in vivo tests are required to ascertain fully their medicinal properties and potential toxicity.
\end{abstract}




\section{Article Outline}

1. Introduction

2. Materials and methods

2.1. Collection of plant material and extract preparation

2.2. Antibacterial assay

2.3. Anthelmintic assay

2.4. Brine shrimp lethality assay

3. Results

3.1. Antibacterial activity

3.2. Anthelmintic activity

3.3. Brine shrimp lethality/toxicity

4. Discussion

5. Conclusion

Acknowledgements

References

\section{Introduction}

Ethnoveterinary medicine is a broad field covering people's beliefs, skills, knowledge and practices relating to the care of their animals (McCorkle, 1986). The recent revival of scientific interest in traditional veterinary medicine has followed the well-documented interest in traditional practices in human medicine (Schillhorn van Veen, 1997). In animal health, as in human health, the market in traditional medicines is expanding, and traditional practices are increasingly becoming mainstream (Schillhorn van Veen, 1997). Ethnoveterinary medicine is important in areas of developing countries that lack access to conventional medicines for animal health care, which are often unaffordable to poor rural farmers. A key objective of the scientific study of ethnoveterinary practices is the development and promotion of effective veterinary medicines based on inexpensive locally available plants. While the value of conventional medicines in combating infectious diseases is irrefutable, for common diseases such as mild diarrhoea, skin diseases, intestinal worms, wounds and reproductive disorders, ethnoveterinary medicine may have much to offer (Martin et al., 2001). Drawbacks of traditional medicinal plant remedies include seasonal unavailability of plants, the possibility of ineffective or harmful treatments, uncertain dosages and lack of standardisation (Martin et al., 2001). 
A survey of the use of medicinal plants in cattle by Setswana-speaking people in the Madikwe area of the North West Province of South Africa recorded the use of 45 plant species representing 24 families (Van der Merwe et al., 2001). Ethnoveterinary plant use was widespread in this rural part of the country, and many different plants were used for various ailments. The most commonly treated disorders include diarrhoea, eye inflammation, general gastrointestinal problems, retained placenta, heartwater, internal parasites, coughing, redwater and tick infestation (Van der Merwe et al., 2001). The plant material was traditionally prepared in various ways including infusion, decoction, ground fresh material and sap expressed from fresh material, and in most cases a liquid was orally administered using a bottle.

Based predominantly on the ethnoveterinary use of plants reported by Van der Merwe et al. (2001), as well as on knowledge gathered in unreported studies, plants used to treat infectious disorders were collected and assayed for biological activity in an endeavour to discover those that were highly active and also to validate their traditional use. The plant parts employed to prepare the extracts were those used in traditional ethnoveterinary medicine.

Antibacterial effects against two Gram-positive and two Gram-negative species were investigated using a microdilution assay. Anthelmintic activity was examined using a free-living nematode as a model. Additionally, the extracts were submitted to the brine shrimp lethality assay as activity in this assay has been correlated to pharmacological activity (McLaughlin, 1991).

\section{Materials and methods}

\subsection{Collection of plant material and extract preparation}

Plant material was collected in the northern and eastern parts of South Africa in the summer of 2001/2002. Voucher specimens ${ }^{2}$ were prepared and deposited in the Herbarium of the Onderstepoort Veterinary Institute (Pretoria). The place of collection as well as the known ethnoveterinary use of the plants were recorded.

After drying at room temperature in a well-ventilated room, the plant material was ground to a powder using a Janke and Kunkel mill. Three separate aliquots of $2 \mathrm{~g}$ of parts of each plant were extracted by shaking vigorously for 20 min on a Labotec Model 20.2 shaker with $20 \mathrm{~mL}$ of hexane, methanol or water. The extract was allowed to settle, 
centrifuged at $2000 \mathrm{~g}$ for $10 \mathrm{~min}$ and the supernatant filtered through Whatman No. 1 filter paper. The extracts were dried in a stream of cold air before resuspending in acetone in the case of the organic extracts, and water for aqueous extracts, to a concentration of $100 \mathrm{mg} \mathrm{mL}^{-1}$. In total, 70 extracts were prepared from 17 species ( 24 plants) for bioassay screening against bacteria, nematodes and brine shrimp larvae.

\subsection{Antibacterial assay}

The serial microplate dilution method of Eloff (1998) was used to screen the plant extracts for antibacterial activity. This method allows the determination of the minimal inhibitory concentration (MIC) of each plant extract against each bacterial species by measuring reduction of tetrazolium violet.

The bacteria used in the present study included two Gram-positive bacteria, Enterococcus faecalis (ATCC 29212) and Staphylococcus aureus (ATCC 29213), and two Gramnegative species, Pseudomonas aeruginosa (ATCC 27853) and Escherichia coli (ATCC 35219). The bacterial cultures were incubated in Müller-Hinton (MH) broth overnight at $37{ }^{\circ} \mathrm{C}$ and a $1 \%$ dilution of each culture in fresh $\mathrm{MH}$ broth was prepared prior to use in the microdilution assay. Twofold serial dilutions of plant extract $(100 \mu \mathrm{L})$ were prepared in 96-well microtitre plates, and $100 \mu \mathrm{L}$ of bacterial culture were added to each well. The plates were incubated overnight at $37^{\circ} \mathrm{C}$ and bacterial growth was detected by adding $40 \mu \mathrm{L}$ p-iodonitrotetrazolium violet (INT) (Sigma) to each well. After incubation at $37^{\circ} \mathrm{C}$ for $1 \mathrm{~h}$, INT is reduced to a red formazan by biologically active organisms, in this case, the dividing bacteria. Bacterial growth was shown to be inhibited when the solution in the well remained clear. This concentration was taken to be the minimal inhibitory concentration (MIC). Solvent controls and the standard antibiotic neomycin (Sigma) were included in each experiment.

\subsection{Anthelmintic assay}

Anthelmintic activity of plant extracts was assayed using the free-living nematode Caenorhabditis elegans var. Bristol (N2) following the method of Rasoanaivo and Ratsimamanga-Urverg (1993), modified by McGaw et al. (2000). The nematodes were cultured on nematode growth (NG) agar seeded with E. coli (Brenner, 1974). Approximately 500 nematodes (7-10 day old cultures) in M9 buffer (Brenner, 1974) 
were incubated with $0.5,1$ and $2 \mathrm{mg} \mathrm{mL}^{-1}$ of plant extract for $2 \mathrm{~h}$ at $25^{\circ} \mathrm{C}$ in the dark. The anthelmintic levamisole (Sigma) was used as a positive control, and solvent blanks were included. Using a stereomicroscope, the percentage of living nematodes was estimated.

\subsection{Brine shrimp lethality assay}

The plant extracts were tested against larvae of Artemia salina (brine shrimp) using the method of Solís et al. (1993). Brine shrimp eggs were obtained from a local pet shop and hatched in artificial sea water $\left(3.8 \mathrm{~g} \mathrm{NaCl}\right.$ per $100 \mathrm{~mL}$ distilled $\left.\mathrm{H}_{2} \mathrm{O}\right)$. After $48 \mathrm{~h}$, the phototropic nauplii were collected using a Pasteur pipette. The plant extracts were tested

at concentrations of $0.1,1,2$ and $5 \mathrm{mg} \mathrm{mL}^{-1}$. The plant extract solutions were each placed in two replicate wells of a $96-$ well microtitre plate. A suspension $(100 \mu \mathrm{L}$, containing approximately 10-15 nauplii) was added to each well. Podophyllotoxin (Sigma) was used as a positive control and solvent blanks were included in each assay. The microtitre plate was covered and incubated for $24 \mathrm{~h}$ at room temperature.

The number of dead and live nauplii in each well was counted using a stereomicroscope. If deaths occurred in the solvent controls at the end of the treatment, the percentage of deaths was corrected using Abbott's formula described by Rasoanaivo and Ratsimamanga-Urverg (1993):

\section{Correted montality pereentage : $(m-M) / 5 \times 100$.}

where $m=$ mean percentage of dead larvae in treated tubes, $M=$ mean percentage of dead larvae in solvent controls and $S=$ mean percentage of living larvae in solvent controls.

\section{Results}

\subsection{Antibacterial activity}

The antibacterial activities of the extracts are presented in Table 1. Overall, S. aureus was the most susceptible bacterial species followed by E. faecalis (both Gram-positive), then E. coli and finally P. aeruginosa (Gram-negative). Few extracts displayed activity against both Gram-negative and Gram-positive bacteria. 
Table 1.

Antibacterial, anthelmintic and toxic (brine shrimp lethality) activity of plants used in ethnoveterinary medicine in the northern parts of South Africa

\begin{tabular}{|c|c|c|c|c|c|c|c|c|c|c|c|}
\hline \multirow[t]{2}{*}{ Family and species } & \multirow[t]{2}{*}{$\begin{array}{l}\text { Plant } \\
\text { part }^{\mathrm{a}}\end{array}$} & \multirow[t]{2}{*}{ Extract $^{\mathrm{b}}$} & \multicolumn{5}{|c|}{$\begin{array}{l}\text { Antibacterial activity (MIC in } \\
\left.\mathrm{mg} \mathrm{mL}^{-1}\right)^{\mathrm{c}}\end{array}$} & \multicolumn{3}{|c|}{$\begin{array}{l}\text { Anthelmintic activity } \\
\left(\mathrm{mg} \mathrm{mL}^{-1}\right)\end{array}$} & \multirow[t]{2}{*}{$\begin{array}{l}\text { Toxicity } \\
\left(\mathrm{LC}_{50}\right) \\
\left(\mathrm{mg} \mathrm{mL}^{-1}\right)\end{array}$} \\
\hline & & & E.c. & E.f. & P.a. & S.a. & Average & $0.5^{\mathrm{d}}$ & 1 & 2 & \\
\hline \multicolumn{12}{|l|}{ Anacardiaceae } \\
\hline \multirow[t]{3}{*}{ Rhus lancea L.f. } & BK & $\mathrm{H}$ & $>12.5$ & 6.3 & $>12.5$ & 3.1 & $>8.6$ & $0^{\mathrm{e}}$ & 2 & 2 & $n^{f}$ \\
\hline & & M & $>12.5$ & 0.2 & $>12.5$ & 0.2 & $>6.4$ & 0 & 0 & 1 & $\mathrm{n}$ \\
\hline & & W & $>12.5$ & $>12.5$ & $>12.5$ & $>12.5$ & $>12.5$ & 0 & 0 & 0 & 3.9 \\
\hline \multirow[t]{3}{*}{ Rhus lancea L.f. } & $\mathrm{LF}$ & $\mathrm{H}$ & $>12.5$ & $>12.5$ & $>12.5$ & 3.1 & $>10.2$ & 3 & 3 & 4 & $\mathrm{n}$ \\
\hline & & M & $>12.5$ & 12.5 & $>12.5$ & 0.2 & $>9.4$ & 0 & 0 & 0 & 1.0 \\
\hline & & $\mathrm{W}$ & $>12.5$ & $>12.5$ & $>12.5$ & 12.5 & $>12.5$ & 0 & 0 & 0 & 0.6 \\
\hline Sclerocarya birrea (A. & BK & $\mathrm{H}$ & $>12.5$ & $>12.5$ & $>12.5$ & $>12.5$ & $>12.5$ & 0 & 0 & 1 & $\mathrm{n}$ \\
\hline
\end{tabular}




\begin{tabular}{|c|c|c|c|c|c|c|c|c|c|c|c|}
\hline \multirow[t]{2}{*}{ Family and species } & \multirow[t]{2}{*}{$\begin{array}{l}\text { Plant } \\
\text { part }^{\mathrm{a}}\end{array}$} & \multirow[t]{2}{*}{ Extract $^{b}$} & \multicolumn{5}{|c|}{$\begin{array}{l}\text { Antibacterial activity (MIC in } \\
\left.\mathrm{mg} \mathrm{mL}^{-1}\right)^{\mathrm{c}}\end{array}$} & \multicolumn{3}{|c|}{ 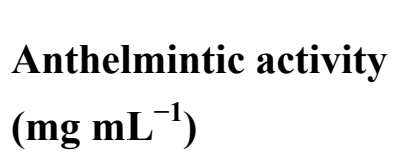 } & \multirow[t]{2}{*}{$\begin{array}{l}\text { Toxicity } \\
\left(\mathrm{LC}_{50}\right) \\
\left(\mathrm{mg} \mathrm{mL}^{-1}\right)\end{array}$} \\
\hline & & & E.c. & E.f. & P.a. & S.a. & Average & $0.5^{\mathrm{d}}$ & 1 & 2 & \\
\hline \multicolumn{12}{|l|}{ Rich.) Hochst } \\
\hline & & M & $>12.5$ & 0.4 & $>12.5$ & 0.1 & $>6.4$ & 1 & 2 & 3 & $\mathrm{n}$ \\
\hline & & $\mathrm{W}$ & $>12.5$ & $>12.5$ & $>12.5$ & $>12.5$ & $>12.5$ & 0 & 0 & 1 & $\mathrm{n}$ \\
\hline \multicolumn{12}{|l|}{ Apocynaceae } \\
\hline $\begin{array}{l}\text { Secamone filiformis (L.f.) } \\
\text { J.H. Ross }\end{array}$ & AP & M & $>12.5$ & 0.8 & $>12.5$ & 1.6 & $>6.9$ & 0 & 0 & 0 & $\mathrm{n}$ \\
\hline \multicolumn{12}{|l|}{ Araliaceae } \\
\hline \multirow[t]{3}{*}{ Cussonia spicata Thunb. } & RT & $\mathrm{H}$ & $>12.5$ & $>12.5$ & $>12.5$ & $>12.5$ & $>12.5$ & 0 & 0 & 0 & $\mathrm{n}$ \\
\hline & & M & 6.3 & 6.3 & $>12.5$ & 6.3 & $>7.9$ & 0 & 0 & 0 & $\mathrm{n}$ \\
\hline & & $\mathrm{W}$ & 12.5 & 12.5 & 12.5 & $>12.5$ & $>12.5$ & 0 & 0 & 0 & 2.6 \\
\hline
\end{tabular}




\begin{tabular}{|c|c|c|c|c|c|c|c|c|c|c|c|}
\hline \multirow[t]{2}{*}{ Family and species } & \multirow[t]{2}{*}{$\begin{array}{l}\text { Plant } \\
\text { part }^{\mathrm{a}}\end{array}$} & \multirow[t]{2}{*}{ Extract $^{b}$} & \multicolumn{5}{|c|}{$\begin{array}{l}\text { Antibacterial activity (MIC in } \\
\left.\mathrm{mg} \mathrm{mL}^{-1}\right)^{\mathrm{c}}\end{array}$} & \multicolumn{3}{|c|}{$\begin{array}{l}\text { Anthelmintic activity } \\
\left(\mathbf{m g ~ m L} \mathbf{L}^{-1}\right)\end{array}$} & \multirow[t]{2}{*}{$\begin{array}{l}\text { Toxicity } \\
\left(\mathrm{LC}_{50}\right) \\
\left(\mathrm{mg} \mathrm{mL}^{-1}\right)\end{array}$} \\
\hline & & & E.c. & E.f. & P.a. & S.a. & Average & $0.5^{\mathrm{d}}$ & 1 & 2 & \\
\hline \multicolumn{12}{|l|}{ Asteraceae } \\
\hline \multirow{3}{*}{$\begin{array}{l}\text { Schkuhria pinnata } \\
\text { (Lam.) Cabrera }\end{array}$} & AP & $\mathrm{H}$ & $>12.5$ & 12.5 & $>12.5$ & 12.5 & $>12.5$ & 0 & 1 & 2 & 3.4 \\
\hline & & M & 12.5 & 6.3 & 12.5 & 1.6 & 8.2 & 0 & 0 & 0 & 2.5 \\
\hline & & $\mathrm{W}$ & $>12.5$ & $>12.5$ & $>12.5$ & $>12.5$ & $>12.5$ & 0 & 0 & 0 & $\mathrm{n}$ \\
\hline \multicolumn{12}{|l|}{ Euphorbiaceae } \\
\hline \multirow[t]{3}{*}{ Ricinus communis L. } & $\mathrm{ST} / \mathrm{LF}$ & $\mathrm{H}$ & 12.5 & 6.3 & $>12.5$ & 3.1 & $>8.6$ & 0 & 1 & 2 & 1.4 \\
\hline & & M & 12.5 & 3.1 & $>12.5$ & 0.8 & $>7.2$ & 0 & 0 & 0 & 1.5 \\
\hline & & $\mathrm{W}$ & $>12.5$ & $>12.5$ & $>12.5$ & $>12.5$ & $>12.5$ & 0 & 1 & 2 & $\mathrm{n}$ \\
\hline & & & & & & & & & & & \\
\hline
\end{tabular}




\begin{tabular}{|c|c|c|c|c|c|c|c|c|c|c|c|}
\hline \multirow[t]{2}{*}{ Family and species } & \multirow[t]{2}{*}{$\begin{array}{l}\text { Plant } \\
\text { part }^{\mathrm{a}}\end{array}$} & \multirow[t]{2}{*}{ Extract $^{b}$} & \multicolumn{5}{|c|}{$\begin{array}{l}\text { Antibacterial activity (MIC in } \\
\left.\mathrm{mg} \mathrm{mL}^{-1}\right)^{\mathrm{c}}\end{array}$} & \multicolumn{3}{|c|}{ 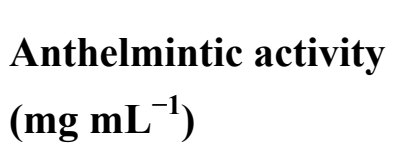 } & \multirow{2}{*}{$\begin{array}{l}\text { Toxicity } \\
\left(\mathrm{LC}_{50}\right) \\
\left(\mathrm{mg} \mathrm{mL}^{-1}\right)\end{array}$} \\
\hline & & & E.c. & E.f. & P.a. & S.a. & Average & $0.5^{\mathrm{d}}$ & 1 & 2 & \\
\hline $\begin{array}{l}\text { Synadenium cupulare } \\
\text { (Boiss.) }\end{array}$ & $\mathrm{ST} / \mathrm{LF}$ & $\mathrm{H}$ & $>12.5$ & 6.3 & $>12.5$ & 6.3 & $>9.4$ & 0 & 0 & 0 & $\mathrm{n}$ \\
\hline \multirow[t]{2}{*}{ L.C. Wheeler } & & M & $>12.5$ & 3.1 & $>12.5$ & 6.3 & $>8.6$ & 0 & 0 & 0 & $\mathrm{n}$ \\
\hline & & $\mathrm{W}$ & $>12.5$ & $>12.5$ & $>12.5$ & $>12.5$ & $>12.5$ & 0 & 0 & 0 & $\mathrm{n}$ \\
\hline \multicolumn{12}{|l|}{ Fabaceae } \\
\hline \multirow[t]{3}{*}{$\begin{array}{l}\text { Pterocarpus angolensis } \\
\text { DC. }\end{array}$} & BK & $\mathrm{H}$ & $>12.5$ & $>12.5$ & $>12.5$ & 12.5 & $>12.5$ & 0 & 0 & 0 & $\mathrm{n}$ \\
\hline & & M & $>12.5$ & $>12.5$ & $>12.5$ & 0.2 & $>9.4$ & 0 & 1 & 1 & $\mathrm{n}$ \\
\hline & & $\mathrm{W}$ & $>12.5$ & $>12.5$ & $>12.5$ & $>12.5$ & $>12.5$ & 0 & 0 & 0 & $\mathrm{n}$ \\
\hline Pterocarpus angolensis & LF & $\mathrm{H}$ & $>12.5$ & 6.3 & $>12.5$ & 3.1 & $>8.6$ & 0 & 0 & 0 & 3.8 \\
\hline
\end{tabular}




\begin{tabular}{|c|c|c|c|c|c|c|c|c|c|c|c|}
\hline \multirow[t]{2}{*}{ Family and species } & \multirow[t]{2}{*}{$\begin{array}{l}\text { Plant } \\
\text { part }^{\mathrm{a}}\end{array}$} & \multirow[t]{2}{*}{ Extract $^{b}$} & \multicolumn{5}{|c|}{$\begin{array}{l}\text { Antibacterial activity (MIC in } \\
\left.\mathrm{mg} \mathrm{mL}^{-1}\right)^{\mathrm{c}}\end{array}$} & \multicolumn{3}{|c|}{ 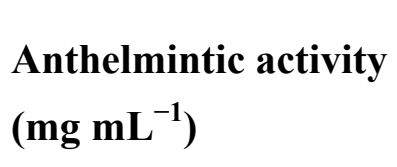 } & \multirow[t]{2}{*}{$\begin{array}{l}\text { Toxicity } \\
\left(\mathrm{LC}_{50}\right) \\
\left(\mathrm{mg} \mathrm{mL}^{-1}\right)\end{array}$} \\
\hline & & & E.c. & E.f. & P.a. & S.a. & Average & $0.5^{\mathrm{d}}$ & 1 & 2 & \\
\hline \multicolumn{12}{|l|}{ DC. } \\
\hline & & M & $>12.5$ & 1.6 & $>12.5$ & 0.4 & $>6.8$ & 0 & 0 & 1 & 3.6 \\
\hline & & $\mathrm{W}$ & $>12.5$ & $>12.5$ & $>12.5$ & $>12.5$ & $>12.5$ & 0 & 0 & 0 & $\mathrm{n}$ \\
\hline \multirow[t]{3}{*}{$\begin{array}{l}\text { Pterocarpus angolensis } \\
\text { DC. }\end{array}$} & BK & $\mathrm{H}$ & $>12.5$ & $>12.5$ & $>12.5$ & 12.5 & $>12.5$ & 0 & 0 & 0 & $\mathrm{n}$ \\
\hline & & M & $>12.5$ & 0.8 & $>12.5$ & 0.2 & $>6.5$ & 0 & 0 & 1 & 1.4 \\
\hline & & $\mathrm{W}$ & $>12.5$ & 1.6 & $>12.5$ & 0.8 & $>6.9$ & 0 & 1 & 1 & $\mathrm{n}$ \\
\hline \multirow{3}{*}{$\begin{array}{l}\text { Pterocarpus angolensis } \\
\text { DC. }\end{array}$} & BK & $\mathrm{H}$ & $>12.5$ & $>12.5$ & $>12.5$ & $>12.5$ & $>12.5$ & 0 & 0 & 0 & $\mathrm{n}$ \\
\hline & & M & $>12.5$ & 1.6 & $>12.5$ & 0.4 & $>6.8$ & 0 & 0 & 1 & 1.5 \\
\hline & & $\mathrm{W}$ & $>12.5$ & 1.6 & $>12.5$ & 1.6 & $>7.1$ & 0 & 1 & 1 & $\mathrm{n}$ \\
\hline
\end{tabular}




\begin{tabular}{|c|c|c|c|c|c|c|c|c|c|c|c|}
\hline \multirow[t]{2}{*}{ Family and species } & \multirow[t]{2}{*}{$\begin{array}{l}\text { Plant } \\
\text { part }^{\text {a }}\end{array}$} & \multirow[t]{2}{*}{ Extract $^{b}$} & \multicolumn{5}{|c|}{$\begin{array}{l}\text { Antibacterial activity (MIC in } \\
\left.\mathrm{mg} \mathrm{mL}^{-1}\right)^{\mathrm{c}}\end{array}$} & \multicolumn{3}{|c|}{ 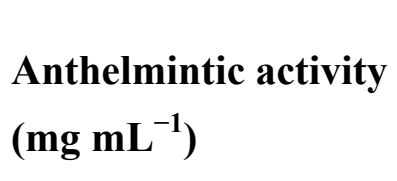 } & \multirow[t]{2}{*}{$\begin{array}{l}\text { Toxicity } \\
\left(\mathrm{LC}_{50}\right) \\
\left(\mathrm{mg} \mathrm{mL}^{-1}\right)\end{array}$} \\
\hline & & & E.c. & E.f. & P.a. & S.a. & Average & $0.5^{\mathrm{d}}$ & 1 & 2 & \\
\hline \multirow{3}{*}{$\begin{array}{l}\text { Schotia brachypetala } \\
\text { Sond. }\end{array}$} & BK & $\mathrm{H}$ & $>12.5$ & $>12.5$ & $>12.5$ & $>12.5$ & $>12.5$ & 0 & 0 & 1 & $\mathrm{n}$ \\
\hline & & M & $>12.5$ & 0.2 & $>12.5$ & 0.1 & $>6.3$ & 0 & 0 & 1 & $\mathrm{n}$ \\
\hline & & $\mathrm{W}$ & $>12.5$ & $>12.5$ & $>12.5$ & 12.5 & $>12.5$ & 0 & 1 & 1 & $\mathrm{n}$ \\
\hline \multirow{3}{*}{$\begin{array}{l}\text { Schotia brachypetala } \\
\text { Sond. }\end{array}$} & LF & $\mathrm{H}$ & 12.5 & 3.1 & 12.5 & 6.3 & 8.6 & 0 & 0 & 1 & 3.3 \\
\hline & & M & $>12.5$ & 0.4 & $>12.5$ & 0.2 & $>6.4$ & 0 & 0 & 0 & $\mathrm{n}$ \\
\hline & & W & $>12.5$ & $>12.5$ & $>12.5$ & 3.1 & $>10.2$ & 0 & 0 & 0 & $\mathrm{n}$ \\
\hline \multicolumn{12}{|l|}{ Pedaliaceae } \\
\hline Dicerocaryum & WP & $\mathrm{H}$ & $>12.5$ & 6.3 & $>12.5$ & 1.6 & $>8.2$ & 0 & 0 & 0 & 0.8 \\
\hline
\end{tabular}




\begin{tabular}{|c|c|c|c|c|c|c|c|c|c|c|c|}
\hline \multirow[t]{2}{*}{ Family and species } & \multirow[t]{2}{*}{$\begin{array}{l}\text { Plant } \\
\text { part }^{\mathrm{a}}\end{array}$} & \multirow[t]{2}{*}{ Extract $^{b}$} & \multicolumn{5}{|c|}{$\begin{array}{l}\text { Antibacterial activity (MIC in } \\
\left.\mathrm{mg} \mathrm{mL}^{-1}\right)^{\mathrm{c}}\end{array}$} & \multicolumn{3}{|c|}{$\begin{array}{l}\text { Anthelmintic activity } \\
\left(\mathrm{mg} \mathrm{mL}^{-1}\right)\end{array}$} & \multirow[t]{2}{*}{$\begin{array}{l}\text { Toxicity } \\
\left(\mathrm{LC}_{50}\right) \\
\left(\mathrm{mg} \mathrm{mL}^{-1}\right)\end{array}$} \\
\hline & & & E.c. & E.f. & P.a. & S.a. & Average & $0.5^{\mathrm{d}}$ & 1 & 2 & \\
\hline \multicolumn{12}{|l|}{$\begin{array}{l}\text { eriocarpum (Decne.) } \\
\text { Abels }\end{array}$} \\
\hline & & M & $>12.5$ & $>12.5$ & $>12.5$ & 1.6 & $>9.8$ & 0 & 0 & 0 & 2.8 \\
\hline & & $\mathrm{W}$ & 6.3 & $>12.5$ & $>12.5$ & 6.3 & $>9.4$ & 0 & 1 & 2 & $\mathrm{n}$ \\
\hline \multicolumn{12}{|l|}{ Rhamnaceae } \\
\hline \multirow[t]{3}{*}{$\begin{array}{l}\text { Berchemia zeyheri } \\
\text { (Sond.) Grubov }\end{array}$} & BK & $\mathrm{H}$ & $>12.5$ & 6.3 & $>12.5$ & 0.8 & $>8.0$ & 1 & 1 & 2 & 3.8 \\
\hline & & M & $>12.5$ & 1.6 & $>12.5$ & 0.2 & $>9.8$ & 0 & 1 & 3 & $\mathrm{n}$ \\
\hline & & W & $>12.5$ & $>12.5$ & $>12.5$ & $>12.5$ & $>12.5$ & 0 & 0 & 0 & $\mathrm{n}$ \\
\hline $\begin{array}{l}\text { Ziziphus mucronata } \\
\text { Willd. }\end{array}$ & BK & $\mathrm{H}$ & $>12.5$ & 12.5 & $>12.5$ & 12.5 & $>12.5$ & 0 & 0 & 0 & $\mathrm{n}$ \\
\hline
\end{tabular}




\begin{tabular}{|c|c|c|c|c|c|c|c|c|c|c|c|}
\hline \multirow[t]{2}{*}{ Family and species } & \multirow[t]{2}{*}{$\begin{array}{l}\text { Plant } \\
\text { part }^{\mathrm{a}}\end{array}$} & \multirow[t]{2}{*}{ Extract $^{\mathrm{b}}$} & \multicolumn{5}{|c|}{$\begin{array}{l}\text { Antibacterial activity (MIC in } \\
\left.\mathrm{mg} \mathrm{mL}^{-1}\right)^{\mathrm{c}}\end{array}$} & \multicolumn{3}{|c|}{ 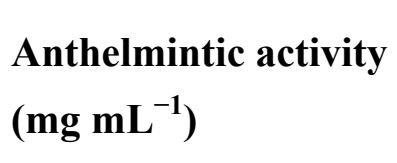 } & \multirow[t]{2}{*}{$\begin{array}{l}\text { Toxicity } \\
\left(\mathrm{LC}_{50}\right) \\
\left(\mathrm{mg} \mathrm{mL}^{-1}\right)\end{array}$} \\
\hline & & & E.c. & E.f. & P.a. & S.a. & Average & $0.5^{\mathrm{d}}$ & 1 & 2 & \\
\hline & & M & $>12.5$ & 3.1 & $>12.5$ & 1.6 & $>7.4$ & 0 & 0 & 0 & $\mathrm{n}$ \\
\hline & & $\mathrm{W}$ & $>12.5$ & $>12.5$ & $>12.5$ & $>12.5$ & $>12.5$ & 0 & 0 & 0 & $\mathrm{n}$ \\
\hline \multirow{3}{*}{$\begin{array}{l}\text { Ziziphus mucronata } \\
\text { Willd. }\end{array}$} & LF & $\mathrm{H}$ & 12.5 & 3.1 & $>12.5$ & 3.1 & $>7.8$ & 0 & 0 & 0 & 0.9 \\
\hline & & M & $>12.5$ & 3.1 & $>12.5$ & 0.2 & $>7.1$ & 0 & 0 & 0 & $\mathrm{n}$ \\
\hline & & $\mathrm{W}$ & $>12.5$ & $>12.5$ & $>12.5$ & $>12.5$ & $>12.5$ & 0 & 0 & 0 & $\mathrm{n}$ \\
\hline \multicolumn{12}{|l|}{ Sapindaceae } \\
\hline \multirow[t]{3}{*}{$\begin{array}{l}\text { Hippobromus pauciflorus } \\
\text { (L.f.) Radlk. }\end{array}$} & AP & $\mathrm{H}$ & $>12.5$ & 6.3 & $>12.5$ & 6.3 & $>9.4$ & 2 & 3 & 4 & $\mathrm{n}$ \\
\hline & & M & $>12.5$ & 3.1 & $>12.5$ & 0.2 & $>7.1$ & 0 & 0 & 0 & $\mathrm{n}$ \\
\hline & & $\mathrm{W}$ & $>12.5$ & $>12.5$ & $>12.5$ & $>12.5$ & $>12.5$ & 0 & 0 & 1 & $\mathrm{n}$ \\
\hline
\end{tabular}




\begin{tabular}{|c|c|c|c|c|c|c|c|c|c|c|c|}
\hline \multirow[t]{2}{*}{ Family and species } & \multirow[t]{2}{*}{$\begin{array}{l}\text { Plant } \\
\text { part }^{\mathrm{a}}\end{array}$} & \multirow[t]{2}{*}{ Extract $^{\mathrm{b}}$} & \multicolumn{5}{|c|}{$\begin{array}{l}\text { Antibacterial activity (MIC in } \\
\left.\mathrm{mg} \mathrm{mL}^{-1}\right)^{\mathrm{c}}\end{array}$} & \multicolumn{3}{|c|}{$\begin{array}{l}\text { Anthelmintic activity } \\
\left(\mathbf{m g ~ m L} \mathbf{L}^{-1}\right)\end{array}$} & \multirow[t]{2}{*}{$\begin{array}{l}\text { Toxicity } \\
\left(\mathrm{LC}_{50}\right) \\
\left(\mathrm{mg} \mathrm{mL}^{-1}\right)\end{array}$} \\
\hline & & & E.c. & E.f. & P.a. & S.a. & Average & $0.5^{\mathrm{d}}$ & 1 & 2 & \\
\hline \multicolumn{12}{|l|}{ Sterculiaceae } \\
\hline \multirow{3}{*}{$\begin{array}{l}\text { Dombeya rotundifolia } \\
\text { (Hochst.) Planch. }\end{array}$} & AP & $\mathrm{H}$ & $>12.5$ & 3.1 & $>12.5$ & 12.5 & $>10.2$ & 0 & 0 & 0 & $\mathrm{n}$ \\
\hline & & M & $>12.5$ & 0.4 & $>12.5$ & 0.4 & $>6.5$ & 0 & 1 & 1 & $\mathrm{n}$ \\
\hline & & $\mathrm{W}$ & $>12.5$ & 1.6 & $>12.5$ & 1.6 & $>7.1$ & 0 & 0 & 0 & $\mathrm{n}$ \\
\hline \multicolumn{12}{|l|}{ Thymelaeaceae } \\
\hline \multirow[t]{3}{*}{ Gnidia capitata L.f. } & RT & $\mathrm{H}$ & $>12.5$ & $>12.5$ & $>12.5$ & $>12.5$ & $>12.5$ & 0 & 0 & 0 & 0.7 \\
\hline & & M & 6.3 & 12.5 & $>12.5$ & 1.6 & $>8.2$ & 0 & 0 & 0 & $\mathrm{n}$ \\
\hline & & $\mathrm{W}$ & $>12.5$ & $>12.5$ & $>12.5$ & $>12.5$ & $>12.5$ & 0 & 0 & 0 & $\mathrm{n}$ \\
\hline & & & & & & & & & & & \\
\hline
\end{tabular}




\begin{tabular}{|c|c|c|c|c|c|c|c|c|c|c|c|}
\hline \multirow[t]{2}{*}{ Family and species } & \multirow[t]{2}{*}{$\begin{array}{l}\text { Plant } \\
\text { part }^{\mathrm{a}}\end{array}$} & \multirow[t]{2}{*}{ Extract $^{b}$} & \multicolumn{5}{|c|}{$\begin{array}{l}\text { Antibacterial activity (MIC in } \\
\left.\mathrm{mg} \mathrm{mL}^{-1}\right)^{\mathrm{c}}\end{array}$} & \multicolumn{3}{|c|}{$\begin{array}{l}\text { Anthelmintic activity } \\
\left(\mathrm{mg} \mathrm{mL}^{-1}\right)\end{array}$} & \multirow[t]{2}{*}{$\begin{array}{l}\text { Toxicity } \\
\left(\mathrm{LC}_{50}\right) \\
\left(\mathrm{mg} \mathrm{mL}^{-1}\right)\end{array}$} \\
\hline & & & E.c. & E.f. & P.a. & S.a. & Average & $0.5^{\mathrm{d}}$ & 1 & 2 & \\
\hline \multicolumn{12}{|l|}{ Urticaceae } \\
\hline \multirow[t]{3}{*}{ Pouzolzia mixta Solms } & $\mathrm{LF}$ & $\mathrm{H}$ & $>12.5$ & $>12.5$ & $>12.5$ & $>12.5$ & $>12.5$ & 0 & 0 & 0 & $\mathrm{n}$ \\
\hline & & M & $>12.5$ & 12.5 & $>12.5$ & 6.3 & $>11.0$ & 0 & 0 & 0 & 5.0 \\
\hline & & $\mathrm{W}$ & $>12.5$ & $>12.5$ & $>12.5$ & $>12.5$ & $>12.5$ & 0 & 0 & 0 & $\mathrm{n}$ \\
\hline \multirow[t]{3}{*}{ Pouzolzia mixta Solms } & ST & $\mathrm{H}$ & $>12.5$ & $>12.5$ & $>12.5$ & $>12.5$ & $>12.5$ & 0 & 0 & 0 & $\mathrm{n}$ \\
\hline & & M & $>12.5$ & 12.5 & $>12.5$ & 12.5 & $>12.5$ & 0 & 0 & 0 & 4.5 \\
\hline & & $\mathrm{W}$ & $>12.5$ & $>12.5$ & $>12.5$ & $>12.5$ & $>12.5$ & 0 & 0 & 0 & $\mathrm{n}$ \\
\hline \multicolumn{12}{|l|}{ Vitaceae } \\
\hline \multirow[t]{2}{*}{ Cissus quadrangularis L. } & ST & $\mathrm{H}$ & $>12.5$ & 6.3 & $>12.5$ & 3.1 & $>8.6$ & 0 & 0 & 0 & $\mathrm{n}$ \\
\hline & & M & 12.5 & 6.3 & 12.5 & 3.1 & 8.6 & 0 & 0 & 0 & 1.3 \\
\hline
\end{tabular}




\begin{tabular}{|c|c|c|c|c|c|c|c|c|c|c|c|}
\hline \multirow[t]{2}{*}{ Family and species } & \multirow[t]{2}{*}{$\begin{array}{l}\text { Plant } \\
\text { part }^{\mathrm{a}}\end{array}$} & \multirow[t]{2}{*}{ Extract $^{\mathrm{b}}$} & \multicolumn{5}{|c|}{$\begin{array}{l}\text { Antibacterial activity (MIC in } \\
\left.\mathrm{mg} \mathrm{mL}^{-1}\right)^{\mathrm{c}}\end{array}$} & \multicolumn{3}{|c|}{$\begin{array}{l}\text { Anthelmintic activity } \\
\left(\mathrm{mg} \mathrm{mL}^{-1}\right)\end{array}$} & \multirow[t]{2}{*}{$\begin{array}{l}\text { Toxicity } \\
\left(\mathrm{LC}_{50}\right) \\
\left(\mathrm{mg} \mathrm{mL}^{-1}\right)\end{array}$} \\
\hline & & & E.c. & E.f. & P.a. & S.a. & Average & $0.5^{\mathrm{d}}$ & 1 & 2 & \\
\hline & & $\mathrm{W}$ & $>12.5$ & 12.5 & $>12.5$ & 6.3 & $>11.0$ & 0 & 0 & 0 & $\mathrm{n}$ \\
\hline Average & & & $>12.23$ & $>8.31$ & $>12.5$ & $>6.69$ & & 0.1 & 0.3 & 0.6 & \\
\hline Neomycin $\left(10^{-3} \times\right)$ & & & 1.56 & 6.25 & 25 & 0.78 & & & & & \\
\hline
\end{tabular}

a Plant part: AP, aerial parts; BK, bark; LF, leaf; RT, root; ST, stem; WP, whole plant.

${ }^{\mathrm{b}}$ Extract: $\mathrm{H}$, hexane; M, methanol; W, water.

${ }^{c}$ Antibacterial activity (MIC): E.c. $=$ Escherichia coli, E.f. $=$ Enterococcus faecalis, P.a. $=$ Pseudomonas aeruginosa,

S.a. $=$ Staphylococcus aureus.

${ }^{\mathrm{d}} \mathrm{mg} \mathrm{mL}^{-1}$ plant extract.

${ }^{\mathrm{e}}$ Scoring system: $0=$ nematode number same as blank (distilled water only), $1=80 \%$ of nematodes alive, $2=70 \%$ of nematodes alive, $3=60 \%$ of nematodes alive, $4=50 \%$ of nematodes alive.

${ }^{\mathrm{f}} \mathrm{n}=$ plant extract has no lethal effect on brine shrimp larvae at concentrations tested. 
In 76/92 analyses, i.e., $83 \%$ of cases, water extracts had no antibacterial activity even at the highest concentration $\left(12.5 \mathrm{mg} \mathrm{mL}^{-1}\right)$. Only one water extract displayed an MIC $<1 \mathrm{mg} \mathrm{mL}^{-1}$ (Pterocarpus angolensis bark against S. aureus) and no aqueous extract exhibited MIC $<1 \mathrm{mg} \mathrm{mL}^{-1}$ against the Gram-negative bacteria. Similarly, only one hexane extract (Berchemia zeyheri bark against $S$. aureus) showed an MIC $<1 \mathrm{mg}$ $\mathrm{mL}^{-1}$ and again no activity was shown against Gram-negative species. In total, 23 extracts $(33 \%)$ exhibited MIC $<1 \mathrm{mg} \mathrm{mL}^{-1}$, of which 21 were methanol extracts, so methanol extracted more antibacterial compounds than did hexane or water.

\subsection{Anthelmintic activity}

Anthelmintic effects of the plant extracts are reported in Table 1. Of the 70 extracts of 24 plants screened in this investigation, 25 (36\%) were active against $C$. elegans. Nine hexane, nine methanol and seven water extracts were active. Hippobromus pauciflorus (hexane extract) demonstrated the highest activity, killing $70 \%$ of nematodes at a concentration of $0.5 \mathrm{mg} \mathrm{mL}^{-1}$ and $50 \%$ of nematodes at $5 \mathrm{mg} \mathrm{mL}^{-1}$. The reference anthelmintic levamisole displayed an $\mathrm{LC}_{50}$ of $10 \mu \mathrm{g} \mathrm{mL}^{-1}$.

\subsection{Brine shrimp lethality/toxicity}

In the brine shrimp lethality assay, $21(30 \%)$ of the extracts showed a degree of activity (Table 1). Seven hexane, eleven methanol and three water extracts showed toxic effects against the brine shrimps. The concentration at which $50 \%$ death of larvae occurred ( $\mathrm{LC}_{50)}$ ranged from 0.6 to $5 \mathrm{mg} \mathrm{mL}^{-1}$ with the aqueous extract of Rhus lancea leaves displaying the highest activity $\left(\mathrm{LC}_{50}=0.6 \mathrm{mg} \mathrm{mL}^{-1}\right)$. The $\mathrm{LC}_{50}$ for podophyllotoxin was $7 \mu \mathrm{g} \mathrm{mL}{ }^{-1}$.

\section{Discussion}

The bacterial strains employed in this study are those recommended for antibacterial activity testing by the United States National Committee for Clinical Laboratory Standards (NCCLS, 1990). Activity was particularly discernible against the Grampositive $S$. aureus and E. faecalis. Gram-positive bacteria are generally more susceptible 
to antimicrobial substances than are Gram-negative species (Vlietinck et al., 1995) owing to differences in the bacterial cell wall structure.

Thirty-three percent of the plant extracts tested had promising antibacterial activity with MIC $<1 \mathrm{mg} \mathrm{mL}^{-1}$. Because $21 / 23$ extracts with $\mathrm{MIC}<1 \mathrm{mg} \mathrm{mL}^{-1}$ were methanol extracts, this indicates that intermediate polarity compounds are active in the antibacterial assays. In most of these cases polar tannins (that would be soluble in water) are probably therefore not involved. Also, the heating and crushing of plant material during the preparation of decoctions or infusions may play a role in liberating active compounds from storage sites in plant tissues. Non-polar compounds may bind non-specifically to macromolecules, such as proteins, in the aqueous solution and dissociate from such binding sites after dosing to be absorbed by the animal.

A number of plant extracts showed weak anthelmintic effects against the free-living nematode C. elegans. After evaluating the anthelmintic properties of extracts of plants used in ethnoveterinary preparations in Kenya, Githiori et al. (2003) reported that none of the plants displayed anthelmintic effects against Heligmosomoides polygyrus in mice. These results support the lack of strong anthelmintic activity reported in the present study. The in vitro anthelmintic assay using C. elegans is simple, cheap and rapid, and supplies an idea of broad-spectrum anthelmintic activity of extracts or compounds. Most commercially available anthelmintics have demonstrable effects on C. elegans, which supported its development as a model for anthelmintic drug screening (Simpkin and Coles, 1981).

There are inevitable limitations of using a free-living nematode to replicate parasitic nematode systems, for example there is no recognition of the complexity of the infectious process (Geary and Thompson, 2001). The in vitro C. elegans assay has not produced valuable new leads since its development (Geary et al., 1999). However, until practical methods for the continuous laboratory culture of parasitic nematodes become available, this whole organism screen, as well as mechanism-based screens, remains an alternative to expensive and time-consuming in vivo assays.

The brine shrimp mortality assay is widely accepted as a convenient probe for potential pharmacological activity in plants (Meyer et al., 1982). Toxic constituents of plant extracts showing lethal effects against the crustacean larvae may elicit interesting effects 
at lower, non-toxic, doses (McLaughlin, 1991). In this investigation the lowest $\mathrm{LC}_{50}$ of $0.55 \mathrm{mg} \mathrm{mL}^{-1}$ compared disappointingly with $\mathrm{LC}_{50}$ of less than $30 \mu \mathrm{g} \mathrm{mL}^{-1}$ for plant extracts against brine shrimp larvae reported by Wanyoike et al. (2004). It would seem that the extracts tested in this study do not possess toxic effects. As seen in Table 1, there were many cases where plant extracts had antibacterial activity but no brine shrimp lethality. There were only two cases where brine shrimp mortality occurred with no corresponding antibacterial activity.

Several correlations in biological activity were noted. Seventeen extracts showed both antibacterial and brine shrimp assay activity. Six extracts showed anthelmintic as well as brine shrimp lethality. Twenty extracts showed anthelmintic and antibacterial activity, while five extracts showed anthelmintic activity but no antibacterial effects. Six extracts showed anthelmintic and brine shrimp assay activity as well as antibacterial activity. Of these, the hexane extract of $B$. zeyheri bark, the methanol extract of $P$. angolensis bark and the stem/leaf hexane extract of Ricinus communis showed good overall activity in the three assays. $P$. angolensis extracts have previously been reported to possess antiinflammatory (Recio et al., 1995) and anti-schistosomal (Ndamba et al., 1994) activity. Related species also have antibacterial activity, namely Pterocarpus osun (Ebi and Ofoefule, 2000) and Pterocarpus indicus (Khan and Omoloso, 2003). R. communis seeds contain highly toxic compounds, the alkaloid ricinine and the lectin ricin (Bruneton, 1995). Leaf infusions of the plant are used in Zulu and Sotho traditional medicine to treat stomach ache, wounds, sores and boils (Watt and Breyer-Brandwijk, 1962 and Hutchings et al., 1996) but active compounds from the leaves do not appear to have been reported. None of the crude extracts showed $\mathrm{LC}_{50}$ or MIC values comparable to those of the reference drugs, but this is not unexpected as the active compound(s) in a crude extract may constitute only a small proportion of the array of compounds in the extract. There are several acknowledged difficulties associated with assessing the validity of traditional practices and remedies. The fact that water extracts did not display any antibacterial activity seems to disprove the local claims that the plants are effective against infectious diseases. However, although the Setswana-speaking people generally apply their remedies as aqueous decoctions or infusions, they do not filter the extracts so that some plant material itself may be ingested by the animal, or applied topically. While 
the results reported provide a useful indication of the in vitro activities of the plants, care should be exercised in extrapolating the results to confirm the efficacy of the local animal healthcare system, as preparation of extracts for the laboratory tests differed from the traditional methods.

Although the necessity to evaluate the safety of traditional medicines is universally accepted, the need for efficacy testing using modern standards is not (Schillhorn van Veen, 1997). When testing medicinal plants for efficacy in a laboratory environment, it should be borne in mind that ethnoveterinary practice is a system (that includes management), which may add to the effectiveness of a particular remedy. However, as some medicines may be toxic, safety testing is required.

Toxicity testing of ethnoveterinary preparations using cell line assays and in vivo tests is essential. Van der Merwe et al. (2001) reported that 36\% of ethnoveterinary remedies were mixtures of different substances perceived to be active, rather than a single constituent. Mixtures may alter the biological activity of plant substances, and activities may be absent when isolated components are used (Bruneton, 1995). Specific methods of preparation and dosage may be needed to allow the active compounds to be absorbed and to reach sufficient concentrations in the animal.

The expanding market for natural plant-based remedies for companion animals cannot be disregarded along with the increasing tendency to minimise the use of antibiotics. The possibility of discovering sources of new remedies for parasitic infestations and bacterial infections in the face of increasing resistance to currently used drugs encourages the evaluation of traditional remedies. In vitro assays may provide a guideline for the selection of highly active plant extracts for subsequent isolation and identification of potentially useful compounds.

Much traditional knowledge will be lost if information is not transferred to younger generations (Van der Merwe et al., 2001), but issues of intellectual property rights add to the complexity of recording and protecting this knowledge. As recommended by Schillhorn van Veen (1997), if traditional systems are used in their correct context and in combination with veterinary practices, particularly in controlling common disorders such as helminth infestations, many of these practices have great potential value. 


\section{Conclusion}

A number of plants used in ethnoveterinary medicine showed promising biological activity in this study, particularly against Gram-positive bacteria. This may indicate that the use of these plants in traditional medicine is of value, especially in terms of topical application where bioavailability and biotransformation are less important. The limitations of the free-living anthelmintic and brine shrimp assays, such as the lack of data correlating activity in the assays with activity in vivo, restrict the value of these techniques. Other methods, such as cell line cytotoxicity assays, may be a more useful indication of toxic effects in vivo.

As toxicity can be associated with pharmacological activity in lower doses, plants containing toxic constituents may have useful biological activities. Further work needs to be focused on isolating biologically active compounds from those plants demonstrating good activity in the initial screening. Members of our group are investigating the biological activity of Rhus spp., Cussonia spp. and Ziziphus mucronata in more detail. An antibacterial compound has been isolated and characterized from Z. mucronata (Moloto, 2004). The use of effective and safe plant extract preparations by rural communities needs to be promoted amongst the people likely to benefit from such applications.

\section{References}

Brenner, 1974 S. Brenner, The genetics of Caenorhabditis elegans, Genetics 77 (1974), pp. 71-94.

Bruneton, 1995 J. Bruneton, Pharmacognosy, Phytochemistry, Medicinal Plants, Intercept, Hampshire (1995).

Ebi and Ofoefule, 2000 G.C. Ebi and S.I. Ofoefule, Antimicrobial activity of Pterocarpus osun stems, Fitoterapia 71 (2000), pp. 433-435.

Eloff, 1998 J.N. Eloff, A sensitive and quick microplate method to determine the minimal inhibitory concentration of plant extracts for bacteria, Planta Medica 64 (1998), pp. $711-$ 713. 
Geary et al., 1999 T.G. Geary, D.P. Thompson and R.D. Klein, Mechanism-based screening: discovery of the next generation of anthelmintics depends upon more basic research, International Journal of Parasitology 29 (1999), pp. 105-112.

Geary and Thompson, 2001 T.G. Geary and D.P. Thompson, Caenorhabditis elegans: how good a model for veterinary parasites?, Veterinary Parasitology 101 (2001), pp. $371-386$.

Githiori et al., 2003 J.B. Githiori, J. Höglund, P.J. Waller and R. Leyden Baker, Evaluation of anthelmintic properties of extracts from some plants used as livestock dewormers by pastoralist and smallholder farmers in Kenya against Heligmosomoides polygyrus infections in mice, Veterinary Parasitology 118 (2003), pp. 215-226. Hutchings et al., 1996 A. Hutchings, A.H. Scott, G. Lewis and A. Cunningham, Zulu Medicinal Plants: An Inventory, University of Natal Press, Pietermaritzburg (1996). Khan and Omoloso, 2003 M.R. Khan and A.D. Omoloso, Antibacterial activity of Pterocarpus indicus, Fitoterapia 74 (2003), pp. 603-605.

Martin et al., 2001 M. Martin, E. Mathias and C.M. McCorkle, Ethnoveterinary Medicine: An Annotated Bibliography of Community Animal Healthcare, ITDG Publishing, London, UK (2001).

McCorkle, 1986 C.M. McCorkle, An introduction to ethnoveterinary research and development, Journal of Ethnobiology 6 (1986), pp. 129-149.

McGaw et al., 2000 L.J. McGaw, A.K. Jäger and J. Van Staden, Antibacterial, anthelmintic and antiamoebic activity in South African medicinal plants, Journal of Ethnopharmacology 72 (2000), pp. 247-263.

McLaughlin, 1991 J.C. McLaughlin, Crown gall tumours on potato discs and brine shrimp lethality: two simple bioassays for higher plant screening. In: K. Hostettman, Editor, Methods in Biochemistry, Assays for Bioactivity vol. 6, Academic Press, London (1991), pp. 1-32.

Meyer et al., 1982 B.N. Meyer, N.R. Ferrigni, J.E. Putnam, L.B. Jacobsen, D.E. Nichols and J.L. McLaughlin, Brine shrimp: a convenient general bioassay for active plant constituents, Journal of Medicinal Plant Research 45 (1982), pp. 31-34. 
Moloto, 2004 Moloto, M.P., 2004. Isolation and characterization of antibacterial, anthelmintic, antioxidant and cytotoxic compounds present in Ziziphus mucronata. MSc thesis, Medical University of Southern Africa, South Africa.

NCCLS, 1990 NCCLS, 1990. The National Committee for Clinical Laboratory

Standards. Performance Standards for Antimicrobial Disk Susceptibility Tests, fourth ed. Approved Standard. NCCLS Document M2-A4. Villanova, PA, USA.

Ndamba et al., 1994 J. Ndamba, N. Nyazema, N. Makaza, C. Anderson and K.C.

Kaondera, Traditional herbal remedies used for the treatment of urinary schistosomiasis in Zimbabwe, Journal of Ethnopharmacology 42 (1994), pp. 125-132.

Rasoanaivo and Ratsimamanga-Urverg, 1993 P. Rasoanaivo and S. RatsimamangaUrverg, Biological Evaluation of Plants with Reference to the Malagasy Flora, Napreca, Madagascar (1993) pp. 9-43, 72-83.

Recio et al., 1995 M.C. Recio, R.M. Giner, S. Manez, J.L. Rios, A. Marston and K. Hostettman, Screening of tropical medicinal plants for anti-inflammatory activity, Phytotherapy Research 9 (1995), pp. 571-574.

Schillhorn van Veen, 1997 T.W. Schillhorn van Veen, Sense or nonsense. Traditional methods of animal parasitic disease control, Veterinary Parasitology 71 (1997), pp. 177194.

Simpkin and Coles, 1981 K.G. Simpkin and G.C. Coles, The use of Caenorhabditis elegans for anthelmintic screening, Journal of Chemical Technology and Biotechnology 31 (1981), pp. 66-69.

Solís et al., 1993 P.N. Solís, C.W. Wright, M.M. Anderson, M.P. Gupta and J.D. Phillipson, A microwell cytotoxicity assay using Artemia salina (brine shrimp), Planta Medica 59 (1993), pp. 250-252.

Van der Merwe et al., 2001 D. Van der Merwe, G.E. Swan and C.J. Botha, Use of ethnoveterinary medicinal plants in cattle by Setswana-speaking people in the Madikwe area of the North West Province of South Africa, Journal of the South African Veterinary Association 72 (2001), pp. 189-196.

Vlietinck et al., 1995 A.J. Vlietinck, L. Van Hoof, J. Totté, A. Lasure, D. Van den Berghe, P.C. Rwangabo and J. Mvukiyumwami, Screening of a hundred Rwandese 
medicinal plants for antimicrobial and antiviral properties, Journal of Ethnopharmacology 46 (1995), pp. 31-47.

Wanyoike et al., 2004 G.N. Wanyoike, S.C. Chhabra, C.C. Lang'at-Thoruwa and S.A.

Omar, Brine shrimp toxicity and antiplasmodial activity of five Kenyan medicinal plants, Journal of Ethnopharmacology 90 (2004), pp. 129-133.

Watt and Breyer-Brandwijk, 1962 J.M. Watt and M.G. Breyer-Brandwijk, The Medicinal and Poisonous Plants of Southern and Eastern Africa (second ed.), Livingstone Ltd., Edinburgh (1962).

Corresponding author. Tel.: +27 12529 8244; fax: +27 125298304 .

${ }^{1}$ Present address: College of Veterinary Medicine, North Carolina State University, 4700 Hillsborough St, Raleigh, NC 27606, USA.

${ }^{2}$ A voucher specimen is a pressed sample of plant material deposited in a herbarium for future reference as it may be examined to verify the identity of the specific plant used in a study. 